\title{
Challenging Ultrasound Diagnoses
}

\author{
Stephen A. Shiver and Matthew Lyon*
}

Medical College of Georgia, 1120 15th Street, AF-2056, Augusta, GA 309812-5800, USA

\begin{abstract}
Bedside ultrasound is a useful tool to the clinician evaluating a possible emergency medical condition. The use of ultrasound by clinicians, from emergency physicians to surgeons to family practice physicians, is increasing. With increased use, unusual or unexpected findings will occur. The clinician-sonographer should be aware of the potential limitations as well as the possible incidental findings inorder to effectively integrate ultrasound into clinical practice. This case series demonstrates three interesting discoveries during the clinical use of ultrasound.
\end{abstract}

Keywords: Bedside ultrasound, Clinical ultrasound, Emergency ultrasound, Liver biopsy, Abdominal hemorrhage, Aortic dissection, Molar pregnancy, Vaginal bleeding.

\section{INTRODUCTION}

The use of bedside ultrasound by clinicians has increased dramatically in the past decade, as it is a useful tool when evaluating a possible emergency medical condition at the patient's bedside. Clinicians using ultrasound are widely variable, from emergency physicians to surgeons to family practice physicians to rheumatologists. With increased use, unusual or unexpected findings will occur. The cliniciansonographer not only needs to have an understanding of the technical aspects of obtaining ultrasound images and clinical integration of the ultrasound findings into patient care, but should also be aware of the potential limitations as well as the possible incidental findings. The following case reports demonstrate the utility as well as possible unexpected findings provided by bedside-clinician-performed ultrasound.

\section{CASE PRESENTATION}

A 46-year-old male presented to the emergency department with a complaint of right upper quadrant abdominal pain. Additional symptoms included lightheadedness and dizziness, which were exacerbated by standing. Past medical history was significant for hepatitis C. Because of progressive elevations in hepatic transaminases, the patient underwent a percutaneous liver biopsy two days prior to presentation in the emergency department.

At the time of initial evaluation, the patient was noted to be normotensive, but with an elevated heart rate of 130 . He was afebrile and in no overt distress. Abdominal examination revealed moderate right upper quadrant tenderness but no peritoneal signs. Intravenous access was achieved, isotonic crystalloid administered, and laboratory studies ordered. Rapid bedside hemoglobin analysis revealed a hemoglobin level of $9.5 \mathrm{~g} / \mathrm{dL}$, a drop of $5 \mathrm{~g} / \mathrm{dL}$ from previously documented levels.

Since a percutaneous liver biopsy was performed two days prior to presentation, concern arose for an iatrogenic

*Address correspondence to this author at the Medical College of Georgia, 1120 15th Street, AF-2056, Augusta, GA 309812-5800, USA; Tel: 706-7214467; Fax: 706-721-7718; E-mail: mlyon@mcg.edu complication. Specifically, it was felt that the patient may be suffering from post biopsy hepatic hemorrhage. An ultrasound credentialed emergency physician performed a bedside ultrasound examination using an Phillips HDI 4000 machine with a curvilinear 5 to $2 \mathrm{MHz}$ transducer. The examination was similar to that used in the Focused Assessment with Sonography for Trauma (FAST) exam. Significant amounts of free fluid were noted in both the hepatorenal and splenorenal recesses (Fig. 1). In addition, the liver was noted to have an unusual sonographic appearance consistent with a large hematoma; much of the right lobe was hypoechoic and heterogeneous in nature with varying levels of internal echoes (Fig. 2).

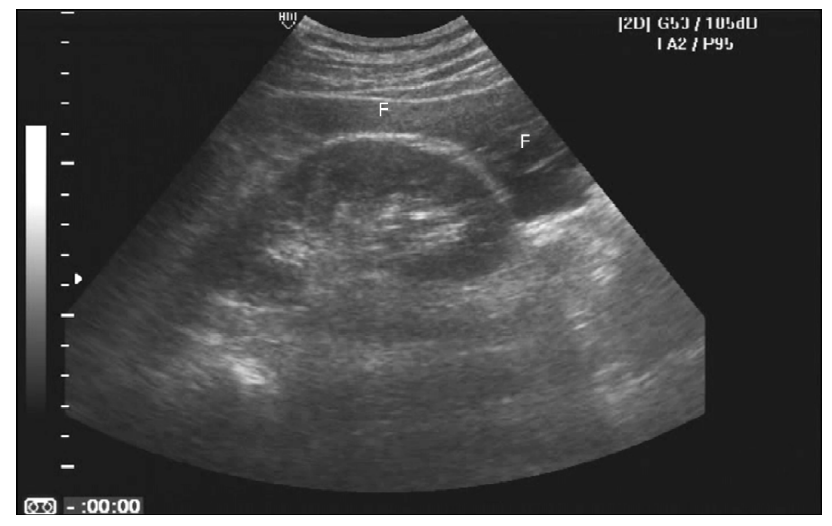

Fig. (1). Free fluid is seen near and inferior pole if the kidney (F: fluid).

Resuscitation was continued and a decision was made to obtain an abdominal computed tomography (CT) scan with intravenous contrast. Though the diagnosis was not in question, the CT scan provided additional information regarding the current state of hemorrhage. CT evaluation showed a massive, predominately subcapsular, liver hematoma with no active extravasation of contrast (Fig. 3). A surgical consultation was obtained and the patient was admitted to the intensive care unit for a period of close observation. The hemoglobin was noted to trend downward, reaching a nadir of 7.5 $\mathrm{g} / \mathrm{dL}$, and, as a result, blood transfusion was required. The 
patient's condition stabilized and no operative intervention was necessary.

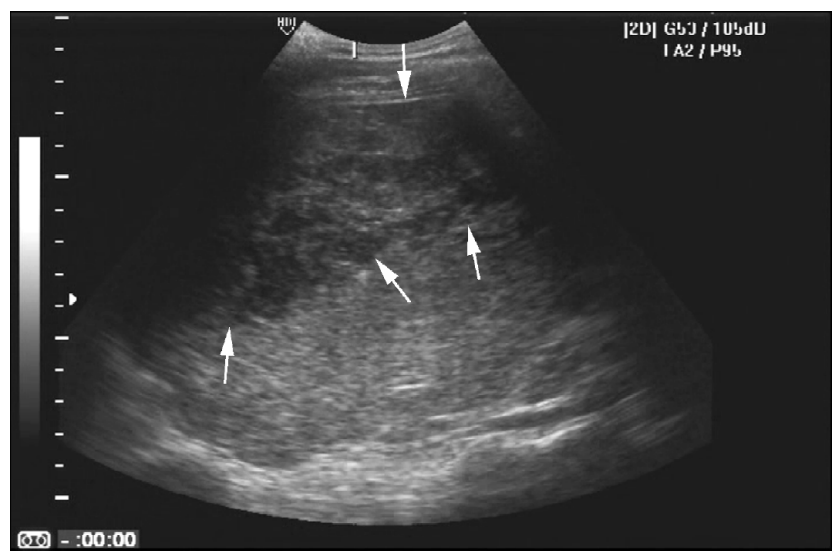

Fig. (2). Heterogenous changes are noted in the liver corresponding to hematoma (arrows).

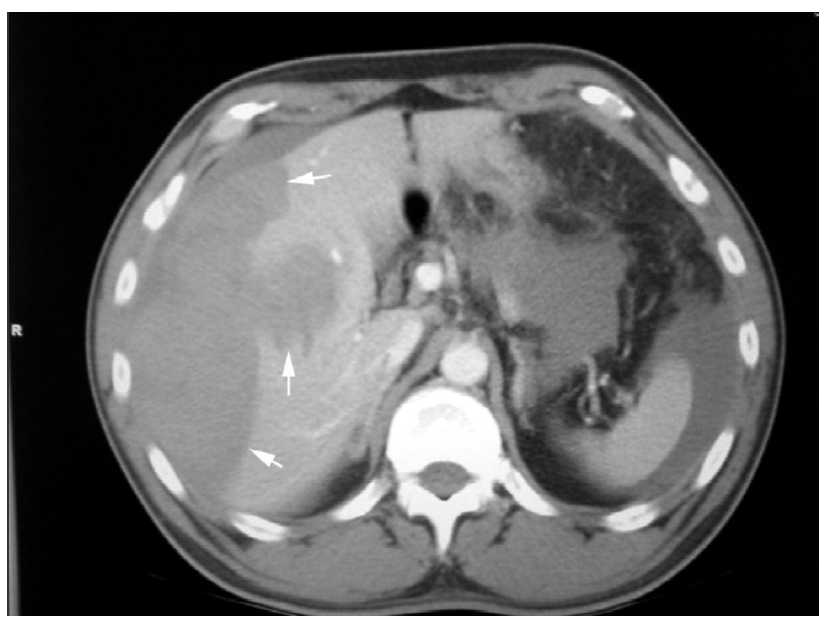

Fig. (3). Large area of subcapsular hematoma is seen (arrows), with no extravasation of contrast noted.

\section{DISCUSSION}

The first known percutaneous liver biopsy was performed 122 years ago by Paul Ehrlich in Germany [1]. Morbidity and mortality were significant in the early experience and the technique did not gain widespread popularity until 1953. It was at that time that Menghini revolutionized the process by introducing a needle that shortened the intrahepatic phase of the procedure [2]. As the safety profile improved, the number of procedures increased and percutaneous biopsy has become the central component in the investigation of hepatic disease. Histological analysis provides important information in a number of disease states including hepatitis of uncertain etiology, neoplasia, post transplant rejection, and chronic hepatitis $\mathrm{C}$ [3].

The statistics on bleeding complications and mortality associated with the procedure have varied in the literature. Bleeding rates of 0.06 to $1.7 \%$ and mortality rates of 0.009 to $0.33 \%$ have been reported [3]. In most series, bleeding complications have been identified by hemodynamic instability or a significant decrease in hemoglobin concentration fol- lowing the procedure. Minor bleeding is likely to be a much more common event.

Ultrasound guidance is often utilized in performing the procedure. However, ultrasound is not routinely employed in the post biopsy period. Some retrospective studies assessing bleeding rates have incorporated information gleaned by post biopsy ultrasound examinations. In such cases, the presence of either intraperitoneal blood or intrahepatic hematoma was considered an indicator for post biopsy hemorrhage [4].

No reports of the utilization of emergency bedside ultrasound in the diagnosis of bleeding complications following percutaneous liver biopsy exist in the literature. Significant bleeding complications will be suspected clinically based on tachycardia, orthostasis, shock, etc. The diagnosis may be confirmed rapidly by bedside ultrasound. Otherwise, diagnosis by computed tomography may add considerable time depending on administration of contrast and transfer time to the computed tomography suite. Expected ultrasound findings in cases of significant hemorrhage include free intraperitoneal fluid and possibly the identification of an intrahepatic or subcapsular hematoma. Identification of a hematoma provides concrete evidence of a bleeding complication. However, patients undergoing percutaneous hepatic biopsy may have ascites and the presence of intraperitoneal fluid must be interpreted within the clinical context.

\section{CASE PRESENTATION}

A 47 year old male with a history of hypertension presented to the emergency department complaining of excruciating abdominal pain. He was hemodynamically stable, but in moderate distress secondary to pain. Physical examination was significant for mild, non-focal abdominal tenderness to palpation and diminished bilateral lower extremity pulses. There was no apparent pulsatile abdominal mass, but abdominal aortic aneurysm was nonetheless in the differential and an emergent bedside ultrasound of the abdominal aorta was performed.

Aneurysmal disease was quickly ruled out as the aortic diameter was found to be $1.9 \mathrm{~cm}$ to $2.1 \mathrm{~cm}$. A floating intimal membrane was visualized, however, which strongly suggested the presence of aortic dissection. An emergent contrast enhanced CT of the chest and abdomen was obtained, which confirmed the diagnosis of a Stanford Type B aortic dissection and correlated well with the ultrasound images. (Fig. 4) Sagittal reconstructions of the CT images further revealed the extent of the dissection. (Fig. 5) Blood pressure was controlled via an intravenous infusion of esmolol and nipride. After an evaluation by cardiothoracic surgery and cardiology, the patient was admitted to the intensive care unit and managed non-operatively.

\section{DISCUSSION}

The incidence of aortic dissection ranges from 5 to 30 cases per million people per year, with the majority of patients dying before presentation to a hospital [5]. The clinical presentation of aortic dissection is easily confused with myocardial infarction, stroke, or other life threatening conditions. Consequently, several studies have shown that the diagnosis of aortic dissection is missed in up to $38 \%$ of patients on initial evaluation [6-8]. Any delay in diagnosis can lead to a higher morbidity and mortality. Thus, it is critical to 


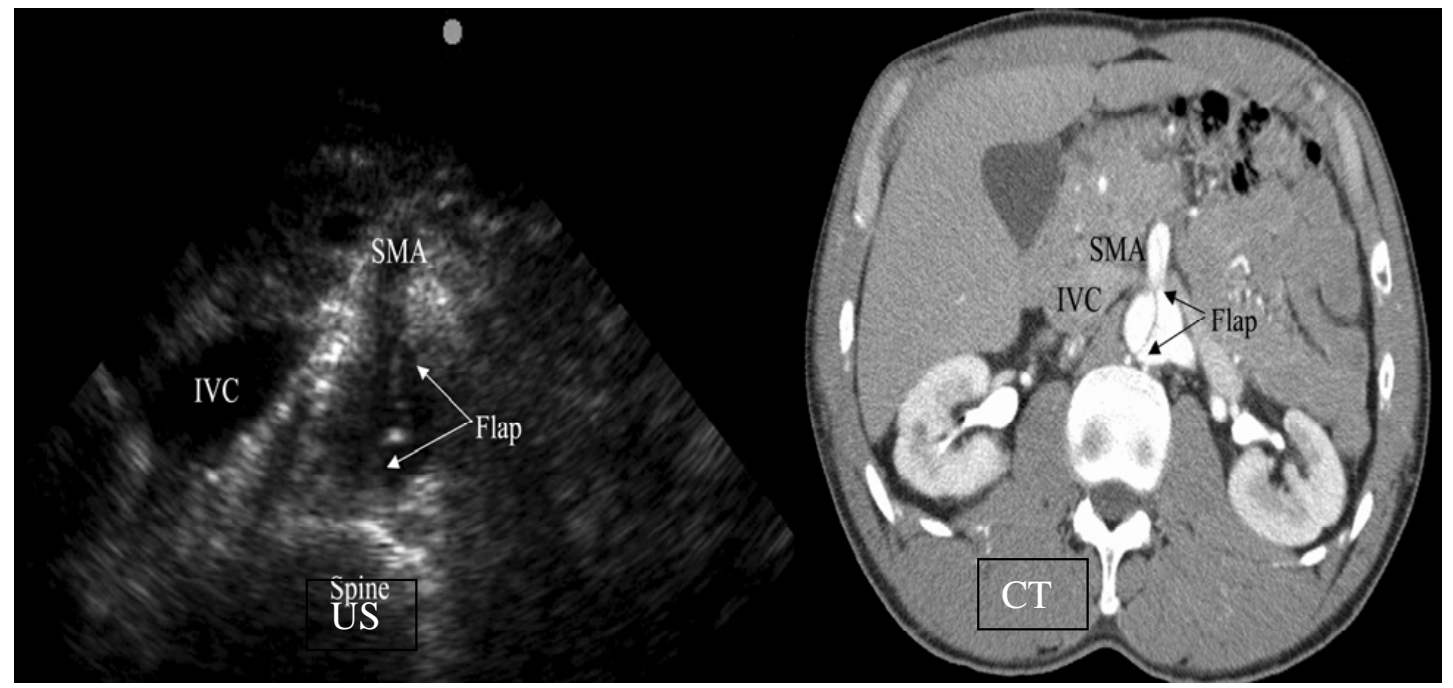

Fig. (4). Transabdominal US and axial CT images at the Level of the superior mesenteric artery demonstrating intimal flap.

maintain a high index of suspicion in establishing the diagnosis.

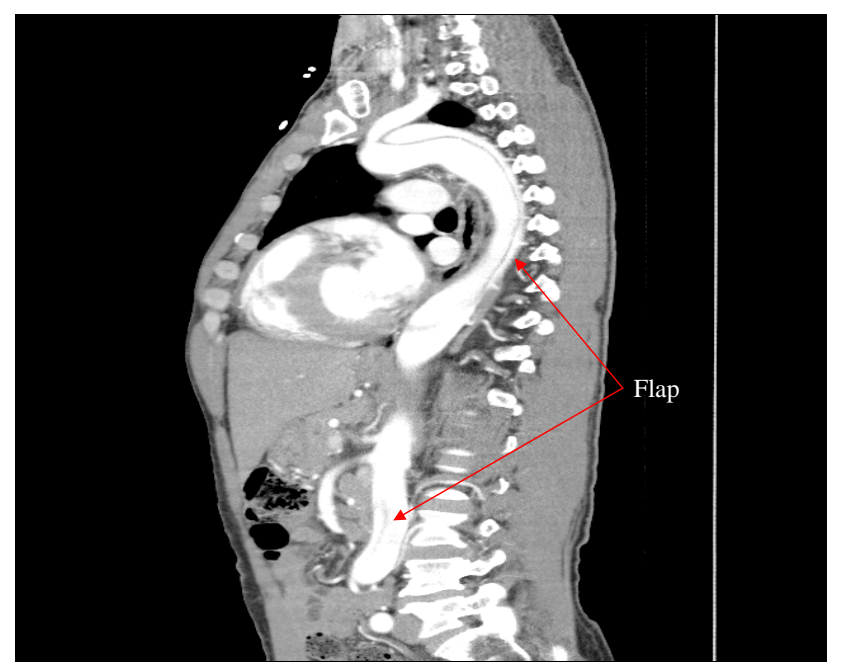

Fig. (5). Sagittal reconstruction of CT images demonstrating intimal flap.

Physical examination for either confirming or ruling out aortic dissection is unreliable. Radiologic imaging, however, is highly sensitive and specific. Computed tomography (CT), magnetic resonance imaging (MRI), angiography, and transesophageal echocardiography (TEE) represent the most commonly utilized and most sensitive diagnostic studies [5].

Acute aortic dissection is frequently fatal, with the mortality rate approaching $1 \%$ per hour during the first 48 hours [9]. If it remains unrecognized and untreated, aortic dissection results in $90 \%$ mortality within the first 3 months, usually due to acute aortic insufficiency, major branch vessel occlusion, or rupture [10]. Expeditious diagnosis is obviously critically important.

Angiography has historically been the most widely used diagnostic modality and is often preferred by surgeons prior to repair. It can differentiate the true and false lumen, identify entry and re-entry sites, demonstrate the anatomy of the major arterial branches, and detect aortic regurgitation. However, angiography is an invasive procedure, utilizes potentially nephrotoxic contrast, and requires time for setup and performance [11]. CT and MRI are also excellent diagnostic options, with sensitivities of 93-100\% and 95-100\%, respectively $[10,12]$. However, both tests usually require transporting an unstable or potentially unstable patient away from the more controlled emergency department environment. MRI, in particular, may be difficult to obtain after hours and in an emergent setting.

Clinicians are increasingly using ultrasound to diagnose and exclude life-threatening pathology in real time. The use of ultrasound in the diagnosis of abdominal aortic aneurysm is common and highly sensitive [13-15]. However, the clinician should be familiar with other diagnoses that may be made or suggested during bedside sonographic evaluations. Though TAS is less sensitive $(<80 \%)$ for the diagnosis of acute aortic dissection than the other more commonly used modalities such as CT, clues to the diagnosis are often present [16-26]. Numerous reports of using TAS in combination with transthoracic echocardiography to diagnose acute aortic dissection exist in the literature [24]. In certain cases, such as the one presented, the diagnosis of dissection can be made or strongly suggested on the basis of TAS alone.

The clinician sonographer must be aware of the sonographic features of acute dissection including the presence of echogenic intimal flaps, a dilated aortic root, and a thickened aortic wall. Discovery of the classically described intimal flap using TAS is pathognomonic for aortic dissection. The intimal flap occurs when blood dissects between the walls of the aorta due to a tear in the innermost layer of the aorta, the intima. This intimal layer can be seen as a thin echogenic linear structure within the aorta and often moves freely with arterial pulsations. This flap can be easily missed or may be mistaken for a sonographic artifact within the aortic lumen. If the membrane is thick or the lumen is thrombosed, the membrane may not move. The aortic lumen may be dilated, but may not be truly aneurysmal. Further, thrombosis of the iliac, celiac, and superior mesenteric arteries may be present and contribute to confusion. 
Determining the extent of the dissection is paramount in the management of an aortic dissection. Dissections involving ascending aorta (Stanford Type A) are associated with a high incidence of mortality due to associated obstruction of vasculature supplying the head, neck, and heart. In addition, ascending arch dissections may extend retrograde and rupture into the pericardium, resulting in immediate tamponade. Dissections limited to the descending aorta (Stanford Type B) may impede blood flow to the mesenteric arteries, renal arteries, spinal cord, lower extremities, etc., and are usually managed non-operatively.

As bedside US becomes more widespread, clinician sonographers are certain to encounter unexpected pathology and must become aware of an ever increasing array of important findings. As illustrated by the case presented, potentially life-threatening processes may be incidentally encountered during scans performed for more common maladies.

\section{CASE PRESENTATION}

A 30 year old female was brought to the emergency department for evaluation of abdominal pain and vaginal bleeding. Her vital signs were unremarkable except for a slightly elevated heart rate of 110. Physical examination revealed mild lower abdominal tenderness to palpation, a small amount of blood in the vaginal vault, and a closed cervical os. Her qualitative urine pregnancy test was positive and her quantitative B-hcg was $133975 \mathrm{mIU} / \mathrm{ml}$. Bedside transvaginal ultrasound was performed (Figs. 6 and 7).

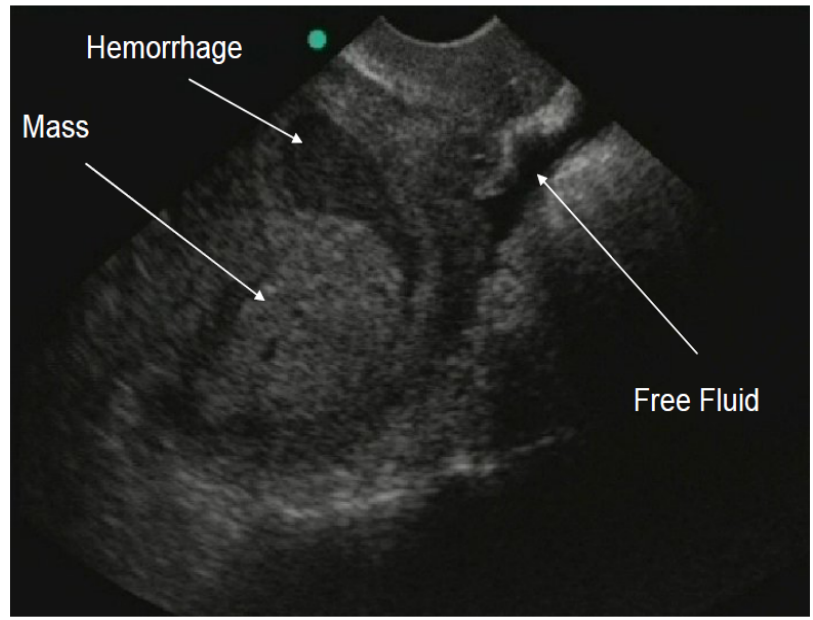

Fig. (6). Long axis view showing intrauterine mass, adjacent hemorrhage, and free fluid.

\section{DISCUSSION}

The transvaginal ultrasound images are consistent with a molar pregnancy. The classic clinical findings associated with molar pregnancy include uterine enlargement, vaginal bleeding, hyperemesis, and elevated B-HCG [26]. The uterine enlargement and magnitude of B-hcg elevation are often more than would be expected given the gestational age. The diagnosis is suspected based on clinical grounds and imaging, but is confirmed by histologic analysis. The classic sonographic appearance of a molar pregnancy is an intrauterine heterogeneous mass with internal hypoechoic regions, previously described as a "snowstorm".

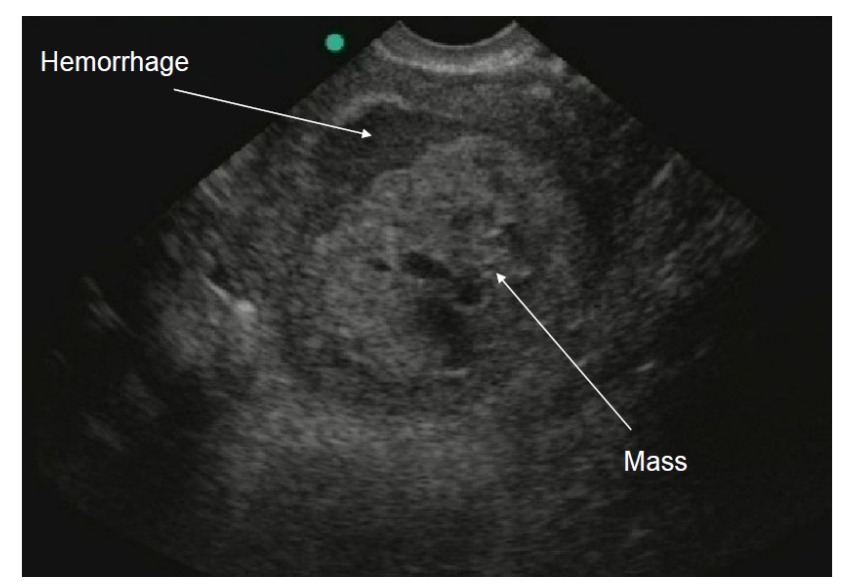

Fig. (7). Short axis view showing intrauterine mass and adjacent hemorrhage.

The patient subsequently underwent suction curretage, with pathology confirming the diagnosis of molar pregnancy. Serial B-HCG levels were monitored postoperatively and were falling appropriately. Malignant gestational trophoblastic disease should be suspected if serum B-hcg levels fail to normalize after uterine evacuation [27].

\section{CONCLUSIONS}

These cases represent unusual findings for the clinician sonographer. While unusual, the sonographer must have knowledge of rare clinical entities. Ultrasound is an excellent modality for detecting free abdominal fluid, but cannot distinguish blood from peritoneal fluid or where the blood is emanating from. Ultrasound is also very useful in the diagnosis of molar pregnancy. While rare, the appearance on ultrasound is characteristic. While not the modality of choice, transabdominal and transthoracic ultrasound may give an indication to the presence of aortic dissection. A flap in the aorta is highly specific for a vascular dissection.

\section{ACKNOWLEDGEMENTS}

A special thanks to Dr. Michael Blaivas, MD, for providing ultrasound images for case one.

\section{REFERENCES}

[1] Von FFT. Uber den diabetes. Berlin: Hirschwald 1884.

[2] Menghini G. One-second biopsy of the liver - Problems of its clinical application. Gastroenterology 1958; 35: 190-9.

[3] Menghini G. One-second biopsy of the liver - Problems of its clinical application. Gastroenterology 1958; 35: 190-9.

[4] Grant A, Neuberger J. Guidelines on the use of liver biopsy in clinical practice. Gut 1999; 45(IV): IV1-IV11.

[5] Terjung B, Lemnitzer I, Dumoulin FL, et al. Bleeding complications after percutaneous liver biopsy. Digestion 2003; 67: 138-45.

[6] Khan IA. Clinical, diagnostic, and management perspectives of aortic dissection. Chest 2002; 122(1): 311-28.

[7] Spittell PC, Spittell JA Jr, Joyce JW, et al. Clinical features and differential diagnosis of aortic dissection: experience with 236 cases (1980 through 1990). Mayo Clin Proc 1993; 68: 642-51.

[8] Bickerstaff LK, Pairolero PC, Hollier LH, et al. Thoracic aortic aneurysms: a population-based study. Surgery 1982; 92: 1103-8.

[9] Eisenberg MJ, Rice SA, Paraschos A, et al. The clinical spectrum of patients with aneurysms of the ascending aorta. Am Heart $\mathrm{J}$ 1993; 125:1380-5.

[10] Hirst AE, Johns VJ, Kime SW. Dissecting aneurysms of the aorta: a review of 505 cases. Medicine 1958; 37: 217-79. 
[11] Olin J, Fuster V. Acute Aortic Dissection: The need for rapid, accurate, and readily available diagnostic strategies. Arterioscler Thromb Vasc Biol 2003; 23(10): 1721-3.

[12] Chandrasekaran K, Currie PJ. Transesophageal echocardiography in aortic dissection. J Invasive Cardiol 1989; 1: 328.

[13] Kahn I, Nair C. Clinical, Diagnostic, and management perspectives of aortic dissection. Chest 2002; 122(1): 311-28.

[14] Kuhn M, Bonnin RL, Davey MJ, et al. Emergency department ultrasound scanning for abdominal aortic aneurysm: accessible, accurate, and advantageous. Ann Emerg Med 2000; 36: 219-23.

[15] Tayal V, Graf C, Gibbs M. Prospective study of accuracy and outcome of emergency ultrasound for abdominal aortic aneurysm over 2 years. Acad Emerg Med 2003; 10: 867-71.

[16] Costantino TG, Bruno EC, Handly N, et al. Accuracy of emergency medicine ultrasound in the evaluation of abdominal aortic aneurysm. J Emer Med 2005; 29: 455-60.

[17] Granato JE, Dee P, Gibson RS. Utility of two-dimensional echocardiography in suspected ascending aortic dissection. Am J Cardiol 1985; 56:123-9.

[18] Kasper W, Meinetz T, Kersting F, et al. Diagnosis of dissecting aortic aneurysm with suprasternal echocardiography. Am J Cardiol 1978; 42: 291-4.

[19] Victor MF, Mintz GS, Kotler MN, et al. Two-dimensional echocardiographic diagnosis of aortic dissection. Am J Cardiol 1981; 48: 1155-9.
[20] Erbel R, Daniel W, Visser C, et al. Echocardiography in diagnosis of aortic dissection. Lancet 1989; 330: 457-60.

[21] Khandheria BK, Tajik AJ, Taylor CL, et al. Aortic dissection: review of value and limitations of two-dimensional echocardiography in a 6 year experience. J Am Soc Echocardiogr 1989; 2: 17-24.

[22] Thomas EA, Dubbins PA. Duplex ultrasound of the abdominal aorta - a neglected tool in aortic dissection. Clin Radiol 1990; 42: 330-4.

[23] Victor MF, Mintz GS, Kolter MN, et al. 2 dimensional echocardiographic diagnosis of aortic dissection. Am J Cardiol 1981; 48: 1155-9.

[24] Roundaut RP, Billes MA, Gosse P, et al. Accuracy of m-mode 2 dimensional echocardiography in the diagnosis of aortic dissection: an experience with 128 cases. Clin Cardiol 1988; 11: 553-62.

[25] Fojtik JP, Costantino TG, Dean AJ. the diagnosis of aortic dissection by emergency medicine ultrasound. J Emer Med 2007; 32(2): 191-6.

[26] Garner E, Goldstein D, Feltmate C, et al. gestational trophobastic disease. clin obstet gynecol 2007; 50(1): 112-22.

[27] Kohorn EI. The New FIGO 2000 staging and scoring system for gestational trophoblastic disease: Description and critical assessment. Int J Gynecol Cancer 2001; 11(1): 73-7.

Received: January 24, 2010

Revised: June 03, 2010

Accepted: June 03, 2010

(c) Shiver and Lyon; Licensee Bentham Open.

This is an open access article licensed under the terms of the Creative Commons Attribution Non-Commercial License (http://creativecommons.org/licenses/by-nc/3.0/) which permits unrestricted, non-commercial use, distribution and reproduction in any medium, provided the work is properly cited. 